\title{
REVIEW
}

\section{Congenital scoliosis in a neonate: can a neonatologist ignore it?}

\author{
S Jog, S Patole, J Whitehall
}

Postgrad Med J 2002;78:469-472

The frequency of diagnosis of congenital scoliosis in the neonatal period is expected to rise given the increasing survival of high risk neonates in the surfactant era and their frequent exposure to $x$ rays. Considering its significant long term implications a neonatologist cannot afford to ignore the diagnosis of congenital scoliosis in a neonate as close surveillance, early detection, and treatment may prevent/minimise the wide spectrum of potentially serious deformities that can affect the developing spine. The review provides general guidelines to help the neonatologists in counselling the parents and in planning the multidisciplinary follow up for management of congenital scoliosis.

See end of article for authors' affiliations

..................

Correspondence to: Dr Sanjay Patole, C/O A S Pabla, PO Box 333, Joondalup, Western Australia 6027; skpatole@hotmail.com

Submitted

14 November 2001

Accepted 13 May 2002

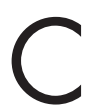
ongenital scoliosis is a lateral curve of the spine due to an imbalance in the longitudinal growth of the spine caused by vertebral anomalies. The deformities caused may be mild, causing subclinical curvatures, or severe, associated with neurological complications, pulmonary restriction, cor pulmonale, and premature death. ${ }^{1}$ The age at diagnosis is reported to vary from the first few days of life to 25 years (average 6 years $).^{2}$ Given the increasing survival of high risk neonates in the surfactant era the frequency of diagnosis of congenital scoliosis in the neonatal period (fig l) is expected to rise in view of their frequent exposure to radiological imaging-a modality that has influenced the care of scoliosis for over 100 years. ${ }^{3}$

As practising neonatologists we recently faced a neonate of 26 weeks' gestation with respiratory distress where congenital scoliosis and a hemivertebra was diagnosed soon after birth on a routine chest radiograph. Repeated queries by the father-a devoted rugby player-about the baby's future ability to play contact sports, need for surgery, and risk of cosmetic deformity ultimately led to an orthopaedic consultation where the need for further follow up was emphasised. Attempting to provide specific answers to the parent's queries we soon realised that the issue of congenital scoliosis in the neonatal period was in "no man's land". A literature search was therefore undertaken to improve the quality of parental counselling while planning the multidisciplinary follow up as a neonatologist. Literature relevant to congenital scoliosis in the neonatal period was searched in Medline, EMBASE, CINAHL, the Cochrane Library, reference lists of review articles, and abstracts published in Pediatric Research. A hand search of paediatric and perinatal journals was also conducted. The following brief review based on this literature search is aimed towards providing general guidelines to help neonatologists in counselling the parents and in planning follow up, which is crucial for management of congenital scoliosis using a multidisciplinary approach. It emphasises the role of a neonatologist in close surveillance, early detection, and management of this congenital anomaly that has a significant impact on the quality of life of patients for years after their discharge from the nursery.

\section{INCIDENCE}

The true incidence of congenital scoliosis in the general population remains unknown as minor deformities often remain undetected. Prenatal detection of an abnormal vertebral body, however, can alert the paediatrician to watch for congenital scoliosis. ${ }^{4}$ About $10 \%$ of cases of structural scoliosis are due to congenital intervertebral or vertebral body abnormalities that cause imbalance in longitudinal growth of the trunk. ${ }^{3}$ Congenital scoliosis is more common in girls than in boys, occurring in the ratio of 2.5:1. Curves occur to left and the right with equal frequency. The incidence of the curve at different levels of

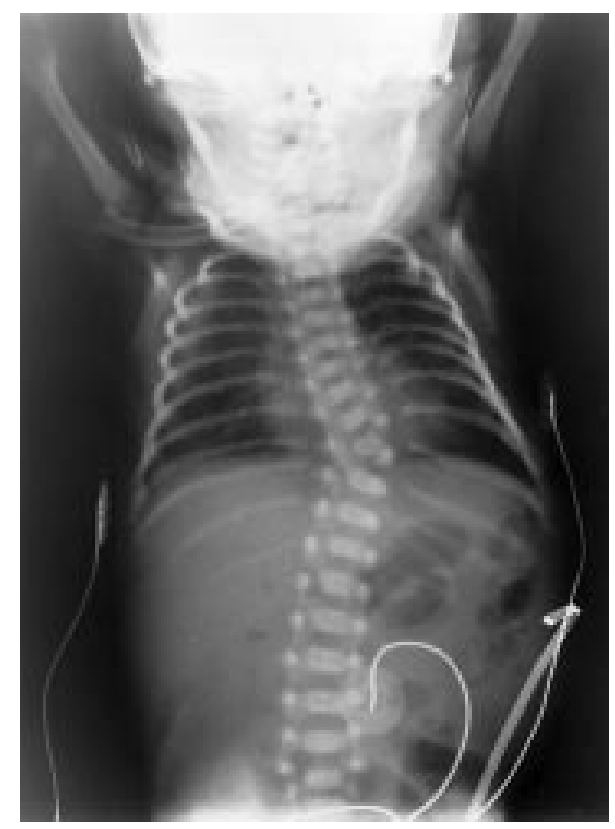

Figure 1 Chest radiograph of a male neonate at 26 weeks' gestation showing lower thoracic scoliosis with concavity to the right, a single hemivertebra at T9, and 11 ribs on the right side. 
the spine is as follows-upper thoracic: $33 \%$, lower thoracic: $31 \%$, thoracolumbar: $20 \%$, lumbar: $11 \%$, and lumbosacral: $5 \%{ }^{5}$

\section{FAMILY HISTORY}

Parental consanguinity and a positive family history (including twin girls and two brothers) of congenital scoliosis have been reported in $40 \%$ and $8 \%$ of patients respectively. ${ }^{2}$ Discordance for congenital scoliosis has also been reported in monozygotic twins, suggesting that it may be acquired in utero rather than genetically determined. ${ }^{6}$

\section{AETIOLOGY}

Failure of formation, segmentation, or a mixture of both, has been proposed as the developmental aetiology of congenital scoliosis. Vertebral anomalies develop during the first six weeks of intrauterine life, when the anatomical pattern of the spine is formed in the mesenchyme. ${ }^{7}$ Hemivertebra is the most common anomaly that causes congenital scoliosis by acting as an enlarging wedge on the affected side of the spine. ${ }^{78}$ The degree of scoliosis produced by hemivertebra depends on their type/site/number/relationship to each other and patient's age. ${ }^{9}$

\section{DIAGNOSIS}

The degree of spinal curvature is measured by traditional Cobb angle determination. ${ }^{10}$ Recently Cobb angle determination has been aided by either computer software programs or a mechanical differential goniometer. ${ }^{112}$

\section{HISTOPATHOLOGY}

A major problem in congenital scoliosis has been reported to relate to the vertebral bodies, which are irregular in size, shape, and position, and to the intervertebral discs, which are always abnormal. ${ }^{13}$ An earlier study reported that the vertebral bone and cartilage as tissues were histologically unremarkable, and that it was their positioning which was irregular. Additionally no molecular abnormality of collagen was reported in the bone, cartilage, or skin. ${ }^{13}$ A subsequent study reported a "normal" percentage of type 1 fibres in the thoracic erector spinae muscles on the concave side but a significantly greater percentage of such fibres on the convex side in patients with congenital scoliosis or scoliosis with early onset. ${ }^{14}$ A relatively recent study has reported reduced proteoglycan and water contents in the cartilage end plate and intervertebral disc, particularly towards the concavity of the curve in congenital scoliosis. ${ }^{15}$ It was suggested that though secondary to the altered loading in the scoliosis, calcification of the cartilage and end plate may be highly significant in the progression of the scoliotic curve. ${ }^{15}$

\section{ASSOCIATED ANOMALIES}

The incidence of spinal cord abnormalities associated with congenital scoliosis is reported to range from $20 \%$ to over $50 \%$ in most of the series. ${ }^{2}{ }^{16}$ Three dimensional computed tomography and magnetic resonance imaging are useful in detecting these abnormalities including tethered cord, syringohydromyelia, Chiari malformation, spinal cord tumour, and the most common intraspinal anomaly, diastematomyelia. ${ }^{16}{ }^{17}$ Among children with congenital spinal anomalies 30\%-60\% are reported to have anomalies at sites other than the spine. The most common of such sites are genitourinary tract, cardiac system, the spinal cord, and the cervical spine. Many are part of VATER syndrome and also incidence of Klippel-Feil syndrome is high. A review of 28 patients with VATER association reported death of three children during the first two years of life. Of the remaining 25 patients, 12 had required orthopaedic procedures for the treatment of congenital scoliosis or deformity of the upper or lower extremities. ${ }^{18}$ Congenital scoliosis has been reported to be associated in $19 \%-70 \%$ of patients with Klippel-Feil syndrome. ${ }^{19-25}$ Duchenne muscular dystrophy best characterises myopathic type of neuromuscular scoliosis whereas cerebral palsy represents the most common form of neuropathic neuromuscular scoliosis. Approximately $90 \%$ of boys with Duchenne muscular dystrophy are reported to develop severe scoliosis by adolescence, not amenable to control by non-surgical means. ${ }^{26}$ In a retrospective review of 45 patients of Duchenne muscular dystrophy no scoliosis was observed in ambulatory patients. However 96\% of the wheelchair bound patients suffered from scoliosis with $96 \%$ having hip flexion or abduction contractures. ${ }^{27}$ In a prospective review of 92 individuals with myotonic dystrophy $47 \%$ of the 17 patients with congenital myotonic dystrophy were noted to have relatively mild, non-progressive scoliosis. Seventy five per cent of these patients also had impaired intellectual and cognitive function, frequently severe ${ }^{28}$ Severe and rapidly progressive congenital scoliosis with multiple joint contractures has also been reported in myopathies like non-progressive central core disease and lipid storage myopathy. ${ }^{29}{ }^{30}$ Recently it has also been reported in association with dysmorphic features and congenital anomalies as in Genoa's syndrome, Mayer-Rokintansky-Küster-Hauser syndrome, and in multiple enchondromatosis with features of dysspondyloenchondromatosis and Maffucci's syndrome. ${ }^{31-33}$

\section{NATURAL HISTORY AND PROGRESSION}

Until 1960 it was thought that congenital scoliosis was non-progressive and thus did not require treatment. McMaster and Ohtsuka and Winter et al refused the theory about the benign nature of congenital scoliosis. ${ }^{734}$ They reviewed a combined total of 485 patients, most until skeletal maturity and found out that almost $75 \%$ of patients required treatment and $84 \%$ of patients who were untreated developed curves greater than 40 degree by maturity. Progression and prognosis were related to type of anomaly and anatomical site of the curve. Children with clinical deformities in the first year of life had the worst prognosis and had early progression. If the curve was present before the patient was 10 years old, it usually increased, in particular during the adolescent growth spurt. ${ }^{7}$ All curves present during infancy and associated with an unsegmented bar deteriorated very rapidly with advancing age as the bar became more ossified. ${ }^{7}$ Similarly other researchers have reported hemivertebra as the most common type and unilateral unsegmented bar with contralateral hemivertebra as the most severe and most progressive pattern of deformity. ${ }^{2}$ Increasingly severe scoliosis may also develop when an asymmetric block vertebra, a wedge vertebra or one or more lateral hemivertebra is present. ${ }^{3}$ Thoracolumbar curves tend to have worst prognosis and the greatest progression followed by lower thoracic curves and upper thoracic curves.

\section{PULMONARY FUNCTION}

Development of restricted pulmonary function is a concern. Curve magnitude directly affects pulmonary function, but the development of restrictive lung function occurs only as the curve approaches 90 degrees. The vital capacity of patients with congenital scoliosis has been compared with those with idiopathic scoliosis. ${ }^{35}$ For any given Cobb angle the loss in vital capacity was approximately $15 \%$ greater in congenital compared with idiopathic scoliosis. This greater impairment in lung function in congenital scoliosis has been proposed to be due to the associated rib deformity or to an underlying lung anomaly. ${ }^{36}$ Vital capacity screening has been recommended for patients with severe curves. A full spirometry work-up is recommended if surgery is planned for those with a vital capacity $<60 \%$ of normal. ${ }^{36}$ 


\section{COSMETIC DEFORMITY}

Cosmetic deformity occurs with most curves. ${ }^{7}$ With upper thoracic curves, elevation of the shoulder on the convexity of the curve, with tilting of the head into the concavity, may be seen. Curves $>30$ degrees may produce an unacceptable deformity, especially in girls. Structural congenital curves tend to have only a mild rotational component, producing only a mild hump. Unbalanced curves in the lower thoracic and lower lumbar region produce a pelvic obliquity with apparent shortening of the leg on the concave side of the curve. Difficulties in ambulation and balance may occur due to the tilting of the trunk away from the apex of the curve. ${ }^{7}$

\section{NON-SURGICAL TREATMENT}

Bracing can treat $<10 \%$ of cases of congenital scoliosis. ${ }^{37}$ However the recommended duration and effects of wearing braces remain controversial. A brace may not arrest or correct the development of curve, but may slow progression and maintain flexibility, allowing surgery at a later stage. ${ }^{37}$

\section{SURGICAL TREATMENT}

Congenital scoliosis is often rigid and correction can be difficult. The goal of surgery is to arrest progression of the curve and correct the deformity to the extent possible. Ideally, surgery is indicated early in curves with rapid progression before they become severe and fixed..$^{37}$ Of the various options available (for example, combined anterior and posterior fusion, resection of hemivertebra) posterior spinal fusion is the oldest and safest spinal procedure and continues to be the gold standard..$^{37}$ Anterior and posterior hemiarthrodesis and hemiepiphyseodesis are reported to be most effective in cases of hemivertebra. ${ }^{37}$ Anterior and posterior arthrodesis (preferably in the first year of life) is also recommended by some authors as a prophylaxis in all midthoracic, thoracolumbar, and lumbar curves as surgical correction after 5 years of age was not successful in their patients. ${ }^{38}$ Semisegmented and incarcerated hemivertebra usually do not require treatment. Fully segmented non-incarcerated hemivertebra may require prophylactic treatment to prevent significant deformity. ${ }^{9}$

It is important to note that the current recommendations for timing and types of options (surgical or non-surgical) for management of congenital scoliosis are not based on randomised controlled trials.

\section{ROLE OF A NEONATOLOGIST}

(1) Apart from recording the demographics a detailed birth, family, and social history should be obtained followed by a thorough clinical, especially neurological, examination.

(2) The nature of the problem and the need for investigations like echocardiography, abdominal/renal/cranial ultrasonographic studies, computed tomography, and magnetic resonance imaging at appropriate times needs to be discussed with the parents as associated malformations can have a serious impact on the outcome (for example, tethered spinal cord).

(3) It is important to emphasise technical difficulties. For example vertebral anomalies may not be recognised radiologically until the neonate is 3-4 years old due to lack of ossification. Similarly sharing the information that hemivertebrae (if diagnosed) could be seen radiologically before the age of 2 years, but as the deformity progresses they could get obscured due to progression of the curve will help the parents to appreciate the need for follow up.

(4) Implications of the site and severity of the spinal curve and the inconstant rate of deterioration of the curve with tendency to deteriorate during time of growth spurts need to be emphasised. This will also be a pointer to the fact that a prolonged follow up is needed. The possibility of need for surgical correction at an early age has to be mentioned in the presence of vertebral anomalies like unilateral unsegmented bar with contralateral hemivertebra.

(5) Given the complexity and diversity of the information to be conveyed it is necessary that multiple meetings with parents and supporting family members are required. Being involved in the day-to-day care of their baby a neonatologist is in a unique position to convey such complex information to parents.

(6) Having counselled the parents about the nature, consequences of the problem, and the available therapeutic options it is then important to arrange a meeting between the parents and the orthopaedic surgeon before discharge of the neonate from the nursery if not earlier. The aim of this meeting is to establish contact between the family and the orthopaedic surgeon, the key person in the multidisciplinary management of the problem.

(7) Available evidence indicates that follow up examinations (measurement of spinal curve, pulmonary function, neurological status) are necessary every 12 months until the age of 10 years and then every 6-12 months until the age of 15 years as rapid deterioration of the spinal curve may occur with growth spurts.

\section{CONCLUSION}

Given its significant long term implications a neonatologist cannot afford to ignore the diagnosis of congenital scoliosis in a neonate as close surveillance, early detection, and treatment may prevent/minimise the wide spectrum of potentially serious deformities that can affect the balance of the developing spine. ${ }^{37}$ In depth parental counselling by the neonatologist is crucial for planning multidisciplinary approach in the management of this problem, which has been known since antiquity. ${ }^{39}$ The task, however, is not easy, as the parents often perceive the neonatologist as doing nothing to "fix the problem". Additionally the specific follow up starts long after discharge of the neonate from the nursery and lasts for years when the neonatologist is not on the scene.

\section{Authors' affiliations \\ S Jog, S Patole, J Whitehall, Department of Neonatology, Kirwan} Hospital for Women, Townsville, Queensland, Australia

\section{REFERENCES}

1 Taphdilan M. Paediatric orthopedica. Philadelphia: WB Saunders, 1990: 2196-207.

2 Shahcheraghi GH, Hobbi MH. Patterns and progression in congenital scoliosis. J Pediatr Orthop 1999; 19:766-75.

3 Oestreich AE, Young LW, Poussaint TY. Scoliosis circa 2000: radiologic imaging perspective 1. Diagnosis and pretreatment evaluation. Skeletal Radiol 1998;27:591-605.

4 Benacerraf BR, Green MF, Barss VA. Prenatal sonographic diagnosis of congenital hemivertebra. J Ultrasound Med 1986;5:257-9.

5 Terminology Committee of the Scoliosis Research Society. A glossary of scoliosis terms. Spine 1976;1:67-8.

6 Pool RD. Congenital scoliosis in monozygotic twins. Genetically determined or acquired in utero? J Bone Joint Surg Br 1986:68: 194-6.

7 McMaster MJ, Ohtsuka K. The natural history of congenital scoliosis. J Bone Joint Surg Am 1982;64: 1 128-47.

8 Imaizumi K, Masuno M, Ishii T, et al. Congenital scoliosis (hemivertebra) associated with de novo balanced reciprocal translocation, 46XX, + (13;17)(q34;p 1 1.2). Am J Med Genet 1997;73:244-6.

9 McMaster MJ, David CV. Hemivertebra as a cause of scoliosis. A study of 104 patients. J Bone Joint Surg Br 1986;68:588-95

10 Young LW, Oestreich AE, Goldstein LA. Roentgenology in scoliosis: contribution to evaluation and management. AJR 1970;108:778-95.

11 Robinson EF, Wade WD. Statistical assessment of two methods of measuring scoliosis before treatment. Can Med Assoc J 1983;129:839-41.

12 Bhullar TPS, Portinaro NMA, Benson MKD. The measurement of angular deformity: an extended role for the "cobometer". J Bone Joint Surg Br 1995;77:506-7.

13 Shapiro F, Eyre D. Congenital scoliosis. A histopathologic study. Spine 1981;6:107-17.

14 Bylund $\mathbf{P}$, Jansson E, Dahlberg $E$, et al. Muscle fibre types in thoracic erector spinae muscles. Fiber types in idiopathic and other forms of scoliosis. Clin Orthop 1987;214:222-8. 
15 Roberts S, Menage J, Eisenstein SM. The cartilage end-plate and intervertebral disc in scoliosis: calcification and other sequelae. J Orthop Res 1993;11:747-57

16 Prahinski JR, Polly DW Jr, McHale KA, et al. Occult intraspinal anomalies in congenital scoliosis. J Pediatr Orthop 2000;20:59-63.

17 Bush CH, Kalen V. Three-dimensional computed tomography in the assessment of congenital scoliosis. Skeletal Radiol 1999;28:632-7.

18 Lawhon SM, MacEwen GD, Bunnel WP. Orthopedic aspects of the VATER association. J Bone Joint Surg Am 1986;68:424-9.

19 Theiss SM, Smith MD, Winter RB. The long-term follow up of patients with Klippel-Feil syndrome and congenital scoliosis. Spine 1997;22:1219-22.

20 Thomsen MN, Schneider U, Weber M, et al. Scoliosis and congenital anomalies associated with Klippel-Feil syndrome types I-III. Spine 1997;22:396-401.

21 Chaumien JP, Rigault P, Maroteaux P, et al. The so called Klippel-Feil syndrome and its orthopedic incidences. Rev Chir Orthop Reparatrice Appar Mot 1990;76:30-8.

22 Van Kerckhoven MF, Fabry G. The Klippel-Feil syndrome: a constellation of deformities. Acta Orthop Belg 1989:55:107-18.

23 Winter RB, Moe JH, Lonstein JE. The incidence of Klippel-Feil syndrome in patients with congenital scoliosis and kyphosis. Spine 1884;9:363-6.

24 Hensinger RN, Lang JE, MacEwen GD. Klippel-Feil syndrome: a constellation of associated anomalies. J Bone Joint Surg Am 1974; 56:1246-53

25 Bernard TN Jr, Burke SW Johnston CE 3rd et al Congenital spinal deformities. A review of 47 cases. Orthopedics 1985;8:777-83

26 Sussman M. Duchenne muscular dystrophy. J Am Acad Orthop Surg 2002;10:138-5

27 Furderer S, Hopf C, Zollner J, et al. Scoliosis and hip flexion contractures in Duchenne muscular dystrophy. Z Orthop Ihre Grenzgeb 2000;138:131-5.
28 Johnson ER, Abresch RT, Carter GT, et al. Profiles of neuromuscular diseases. Myotonic dystrophy. Am J Phys Med Rehabil 1995;74:S104-16.

29 Merlini L, Mattutini P, Bongfiglioli S, et al. Non-progressive central core disease with severe congenital scoliosis: a case report. Dev Med Child Neurol 1987:29:106-9.

30 Nogami H, Ogasawara N, Kasai T, et al. Lipid storage myopathy associated with scoliosis and multiple joint contractures. Acta Neuropathol (Berl) 1983;61:305-10.

31 Lapunzina P, Musante G, Pedraza A, et al. Semilobar holoprosencephaly, coronal craniosynostosis, and multiple congenital anomalies: a severe expression of the Genoa syndrome or a newly recognized syndrome? Am J Med Genet 2001;102:258-60.

32 Fisher K, Esham RH, Thorneycroft I. Scoliosis associated with typical Mayer-Rokintansky-Kuster-Hauser syndrome. South Med J 2000;93:243-6.

33 Haga N, Nakamura K, Taniguchi K, et al. Enchondromatosis with features of dysspondyloenchondromatosis and Maffucci syndrome. Clin Dysmorphol 1998;7:65-8.

34 Winter RB, Moe JH, Eilers VE. Congenital scoliosis. A study of 234 patients treated and untreated. J Bone Joint Surg Am 1968;50: 1-15.

35 Owange-Iraka JW, Harrison A, Warner JO. Lung function in congenital and idiopathic scoliosis. Eur J Pediatr 1984;142:198-200.

36 Muirhead A, Conner AN. The assessment of lung function in children with scoliosis. J Bone Joint Surg Br 1985;67:699-702

37 Jaskwhich D, Ali RM, Patel TC, et al. Congenital scoliosis. Curr Opin Pediatr 2000;1 12:61-6.

38 McMaster MJ. Congenital scoliosis caused by a unilateral failure of vertebral segmentation with contralateral hemivertebrae. Spine 1998:23:998-1005.

39 Sanan A, Rengachary SS. The history of spinal biomechanics. Neurosurgery 1996;39:657-69.

IMAGES IN MEDICINE.

\section{Superior lumbar hernia of Grynfellt}

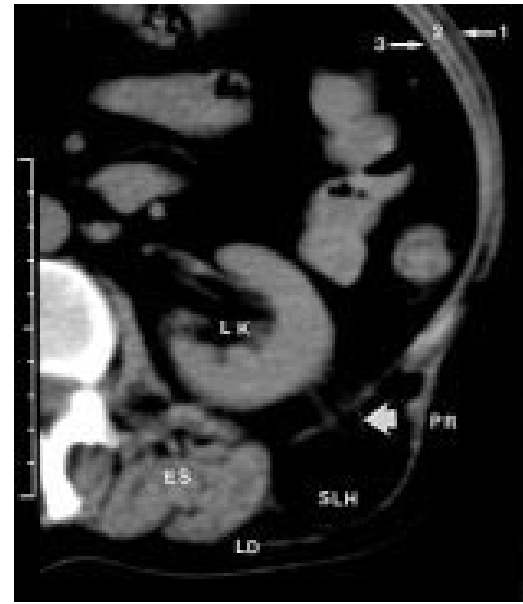

Figure 1 Abdominal computed tomogram showing superior lumbar hernia (SLH). LK, left kidney; PR, perirenal fat passing through transversalis aponeurosis to form SLH (arrowed); ES, erector spinae; LD, latissimus dorsi. (1) External oblique, (2) internal oblique, (3) transversus abdominis.
44 year old woman was investigated for a soft, non-tender, reducible swelling, measuring $5 \mathrm{~cm}$ in diameter in her left lumbar region. There was no cough impulse. An abdominal computed tomogram revealed a superior lumbar hernia, first described by Grynfellt. The hernial content was retroperitoneal fat from around left kidney, which had prolapsed through the transversalis aponeurosis. The external oblique (1), internal oblique (2), and the transversus abdominis (3) lie anteriorly (see fig 1). The erector spinae (ES) forms the posterior boundary. The superior border is formed by the 12 th rib and the iliac crest forms the inferior border. The defect was repaired using interrupted non-absorbable sutures after retracting the latissimus dorsi. The patient has been followed up for a period of 16 weeks and there has been no recurrence.

Superior lumbar hernia is rare and may easily be misdiagnosed as a lipoma if a strong index of suspicion is not kept.
A Pitale

V Laughlin

Lagan Valley Hospital, Hillsborough Road, Lisburn, Co Antrim BT28 1.JP, UK; ashishpitale@lycos.com 